\title{
TDDFT Potential Energy Functions for Excited State Intramolecular Proton Transfer of Salicylic Acid, 3-Aminosalicylic Acid, 5-Aminosalicylic Acid, and 5-Methoxysalicylic Acid
}

\author{
Sungwoo Jang, Sung II Jin, and Chan Ryang Park" \\ Deparment of Chemistry, Kookmin University, Seoul 136-702, Korea. "E-mail crpark@kookminac.kr \\ Received Augtst 9, 2007
}

\begin{abstract}
We report the application of time-dependent density functional theory (TDDFT) to the calculation of potential energy profile relevant to the excited state intramolecular proton transfer (ESIPT) processes in title molecules. The TDDFT single point energy calculations along the reaction path have been performed using the CIS optimized structure in the excited state. In addition to the Stokes shifts, the transition energies including absorption, fluorescence, and 0-0 transition are estimated from the TDDFT potential energy profiles along the proton transfer coordinate. The excited state TDDFT potential energy profile of SA and 3ASA resulted in very flat function of the $\mathrm{OH}$ distance in the range $\mathrm{R}_{\mathrm{OII}}=1.0-1.6 \AA$, in contrast to the relatively deep single minimum function in the ground state. Furthennore, we obtained very shallow double minima in the excited state potential energy profile of SA and 3ASA in contrast to the single minimum observed in the previous work. The change of potential energy protile along the reaction path induced by the substitution of electron donating groups $\left(-\mathrm{NH}_{2}\right.$ and $\left.-\mathrm{OCH}_{3}\right)$ at different sites has been investigated. Substitution at para position with respect to the phenolic $\mathrm{OH}$ group showed strong suppression of excited state proton dislocation compared with unsubstitued SA, while substitution at ortho position hardly affected the shape of the ESIPT curve. The TDDFT results are discussed in comparison with those of CASPT2 method.
\end{abstract}

Key Words : Intramolecular proton transfer, ESIPT, GSIPT, TDDFT, Salicylic acid

\section{Introduction}

Since the first work on methyl salicylate (MS) done by Weller,' the excited-state intramolecular proton transfer (ESIPT) reactions, or the excited-state intramolecular hydrogen transfer (ESIHT) reactions, of MS and its derivatives have attracted a lot of attention in both theoretical and experimental aspects. Due to the fact that the proton transfer processes are known to play an important role in the related fields of chemistry and biochemistry, many photophysical and photochemical investigations on the analogous molecules showing ESIPT phenomena have been done as well as salicylic acid (SA) and its derivatives. ${ }^{1-3}$ Those molecules commonly have a strong intramolecular hydrogen bond between the phenolic hydroxyl group and the neighboring carbonyl oxygen that act as a proton donor and acceptor, respectively. As a result of the acid and base characters of two moieties inside the same molecule, the system forms, so called, a $\mathrm{H}$-chelate ring in the ground electronic state $\left(\mathrm{S}_{0}\right)$. A significant enhancement of the intramolecular hydrogen bond strength in the first electronic excited state $\left(\mathrm{S}_{1}\right)$ and the resulting $\mathrm{H}$-atom transfer comprise the main idea of ESIPT. The photo-induced enol-keto tautormerism in the $S_{1}$ state have lead to an idea of a double minimum potential along the reaction coordinate in the excited electronic state as originally interpreted by Weller. ${ }^{1-3}$ In other words, such molecules exhibit large Stokes shifts in the emission spectra upon photoexcitation to the first excited electronic state, and this Stokes shifted fluorescence has been interpreted as the typical phenomena of ESIPT.
Depending on the shape of the potential energy surface and the barrier heights along the relevant reaction coordinates as well as the magnitude of excitation energy, the ESIPT processes could show a wide range of time scales, ranging from femtoseconds to the microsecond regime. Theoretical considerations on the ESIPT reactions involve the quantum mechanical proton tunneling through a barrier separating two potential minima associated with enol-keto tautomers, while the intramolecular vibrational relaxation (IVR) is considered for the description of a barrierless single minimum potential energy surface. Furthenmore, many laser spectroscopic investigations including fluorescence excitation, dispersed fluorescence, resonance enhanced multiphoton ionization, UV/IR double resonance spectroscopy as well as the femtosecond real-time probing of the related systems in supersonic jets have been performed to elucidate the nature of the ESIPT processes. ${ }^{+10}$ In addition to the conjugation and substituent effects, microsolvation and selfsolvation effects on the excited state intramolecular proton transfer reactions have also been studied both theoretically and experimentally by many research groups. ${ }^{11-16}$

For this historical reason, SA and its derivatives are among the most extensively studied molecules as far as the ESIPT process is concened. Traditionally, the large Stokes shifts in the emission spectra have been attributed to the double minimum potential associating the enol-keto tautomerism along the reaction coordinate in the $\mathrm{S}_{1}$ state. ${ }^{+.8 .9}$ which has been supported by the early stage semiempirical calculations for the potential energy function. ${ }^{16-18}$ Recent experimental and theoretical studies on the SA and its 
derivatives, however, strongly reveal that those molecules involve a single minimum potential for both $\mathrm{S}_{0}$ and $\mathrm{S}_{1}$ states instead of double minima. ${ }^{11,12,19.20}$ According to these results, the large Stokes shifts in the fluorescence spectra are now interpreted in terms of the large structural change in the $\mathrm{S}_{1}$ state mostly through the extensive structural relaxation of the $\mathrm{H}$-chelate ring. In this interpretation, the displacement of the phenolic hydrogen atom along the $\mathrm{O} 1 \mathrm{Hl}$ bond (see Fig. 1 ) is not large enough $(-0.15-0.2 \AA)$ to transfer the proton to the carbonyl oxygen. In addition to this, the spectral evidences that many vibrational modes are active in the optical spectra of SA and its derivatives indicate that the ESIPT process associated with these molecules are attributed to the collective motions of the ring atoms rather than the local geometrical change solely due to hydroxyl proton transfer. In this regard, many authors use a terminology "excited state proton dislocation", 11.12.19.20 which seems to be more appropriate than the conventionally used terminology "excited state proton transfer" for SA and its derivatives.

Recently, diverse and quite sophisticated calculation methods of electronic structure theory have been employed to characterize the potential energy surfaces of the excited electronic states along the reaction path and to calculate the transition energies quite accurately for even fairly large-size molecules. The double minimum potential and high barriers predicted in the semiempirical methods turned out to be due to the neglect of the dynamical electron correlation effects. The $a b$ initio complete-active-space self-consistent-field (CASSCF) method with second-order perturbation theory (CASPT2) have been applied to the calculation of the potential energy function of the lowest excited singlet state $\left(\pi \pi^{*}\right)$ of $a$-hydroxybenzaldehyde (OHBA), and the $\pi \pi^{\circ}$ potential function along the proton transfer reaction coordinate tumed out to have a single minimum with no barrier. ${ }^{1920}$ Another related study on the excited singlet state of $o$ hydroxyacetophenon (OHAP) also reported a single minimum along the proton transfer coordinate after electron correlation is added, whereas the calculations at the $a b$ initio SCF and CIS levels showed two minima corresponding to two tautomeric forms in the $S_{1}$ state. ${ }^{21}$ The same method have been employed to the study of the excited-state intramolecular proton transfer in SA by Sobolewski and Doncke, ${ }^{19.20}$ where $a b$ initio calculations based on multireference perturbation theory (CASPT2) have been performed upon the CIS optimized geometry of SA to construct the potential energy function of the $S_{1}\left(\pi \pi^{3}\right)$ state along the reaction path for the ESIPT process. According to the results of Sobolewski and Domcke, the importance of proper inclusion of dynamical electron correlation effects in the calculation of potential energy function was clearly demonstrated, even though they pointed out that the method might overestimate the stabilization of the potential energy profile in the $S_{1}$ state near the barrier position predicted by CIS method.

Recently, computations using the time-dependent density functional theory (TDDFT) are demonstrated to lead to more accurate predictions of the absorption and fluorescence spectra, and several exploratory calculations on the small and medium sized molecules have been successfully performed. ${ }^{1322-28}$ Also, for the potential energy profiles along the reaction paths, TDDFT method turned out to give at least parallel results compared to those obtained with the CASPT2 method. According to these related results, TDDFT excited state structures, dipole moments, and force constants are now known to be almost as accurate as the corresponding DFT ground state properties, and the same level of accuracy is expected for activation barriers and reaction paths. The TDDFT method has also been successfully applied to the study of intramolecular hydrogen bonding in the first excited state of anthranilic acid (AA) and SA, where the vibrational frequencies and IR intensities as well as the equilivrium geometries have been obtained. ${ }^{29}$ More recently, TDDFT study of the excited state proton transfer in hypoxanthine (HX) have been performed to compute the excitation energies of the $\mathbf{S}_{1} \leftarrow \mathbf{S}_{0}$ transition, where the TDDFT method with the $6-311++\mathrm{G}(\mathrm{d}, \mathrm{p})$ basis set has been employed for the computation of vertical and adiabatic transition energies using the B3LYP and CIS optimized geometries. ${ }^{30}$ The TDDFT excited state potential energy functions of SA, $o$-hydroxybenzaldehyde (OHBA), and 7-hydroxy-1-indanone (7HIN) have been calculated by Sobolewski and Domcke. ${ }^{20}$ In their work, it has been concluded that TDDFT method can be applied to predict reliable shape of the ESIPT energy profiles even though the method gives systematically overestimated values for the transition energies compared to experimental values.

As briefly mentioned above, many theoretical and experimental investigations on SA and its various derivatives have been performed and have focused on the excited state intramolecular proton transfer (ESIPT) processes. In the present work, we applied time-dependent density functional theory (TDDFT) to calculate the potential energy profiles relevant to the ESIPT processes in Salicylic acid (SA), 3aminosalicylic acid (3ASA), 5-aminosalicylic acid (5ASA), and 5-methoxysalicylic acid (5MeOSA). The estimated transition energies for the $S_{1} \leftrightarrow S_{0}$ transitions including absorption, fluorescence, $0-0$ transition, and the Stokes shifts are also presented and compared with the experimental values. The TDDFT results are discussed in comparison with those obtained by CASPT2 method. 5MeOSA has a strong electron donating methoxy group at para position to the phenolic hydroxyl group, and is well known experimentally to suppress the intramolecular proton transfer in the excited state. ${ }^{1.31}$ 3ASA and 5ASA also contain an electron donating amino group at ortho and para position relative to the phenolic hydroxyl group, respectively, and the substitution effects on the ESIPT process have been compared with 5MeOSA as well as SA. The change of potential energy profile along the reaction coordinate induced by the introduction of electron donating groups will be discussed.

\section{Computational Details}

The most stable conformer of SA and its derivatives is presented in Figure 1 with the atomic numbering scheme on 
<smiles>[R2]c1cc([R])c(O[GaH])c(C(=O)O[TlH])c1</smiles>

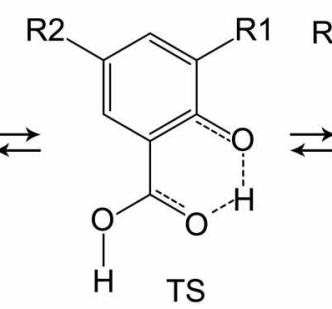<smiles></smiles>

Figure 1. Atomic numbering scheme of the most stable rotamer of $\mathrm{S} \Lambda$ and its derivatives. $\mathrm{S} \Lambda\left(\mathrm{R}_{1}, \mathrm{R}_{2}=\mathrm{H}\right), 3 \wedge \mathrm{S} \wedge\left(\mathrm{R}_{1}=\mathrm{NH}_{2}, \mathrm{R}_{2}=\mathrm{H}\right)$, $5 \Lambda \mathrm{S} \Lambda\left(\mathrm{R}_{1}=\mathrm{H}, \mathrm{R}_{2}=\mathrm{NH}_{2}\right), 5 \mathrm{MeOS} \Lambda\left(\mathrm{R}_{1}=\mathrm{H}, \mathrm{R}_{2}=\mathrm{OCH}_{3}\right)$. The tautomeric reaction scheme relevant to the ESI'T process is shown schematically.

it. SA is known to exist in two forms of planar rotamers. The enol form which has an intramolecular $\mathrm{H}$-bond between $\mathrm{O} 2$ and $\mathrm{H} 1$ (rotamer I) is more stable by about $4-5 \mathrm{kcal} /$ mole than the other enol form which has a H-bond between $\mathrm{O} 3$ and $\mathrm{H} 1$ (rotamer $\Pi$ ), where the carboxyl group is rotated by $180^{\circ}$ with respect to the ring. 5ASA and 5MeOSA have an amino group and a methoxy group at $\mathrm{C} 5$ position, respectively, i.e., para position with respect to the phenolic hydroxyl group. On the other hand, 3ASA contains an amino group at $\mathrm{C} 3$ position, which is ortho to the phenolic hydroxyl group. The enol-keto tautomeric reaction scheme relevant to the excited state intramolecular proton transfer (ESIPT) process is also illustrated schematically in Figure I. The optimizations of the equilibrium geometry in the ground state were carried out using B3LYP/6-31G(d,p) and MP2/6$31 \mathrm{G}(\mathrm{d}, \mathrm{p})$ methods. For the geometry optimization of the excited singlet state, the CIS/6-31G(d,p) method was used. For all the computational methods, the split-valence Gaussian basis set with polarization functions, $6-31 \mathrm{G}(\mathrm{d}, \mathrm{p})$, was employed. The stability of the optimized geometries has been verified by the calculation of Hessian that gives all vibrational frequencies of real numbers. All computations presented here have been performed using the Gaussian 03 series of program. ${ }^{37}$

In dealing with the proton transfer process in the enol-keto tautormeric reaction, defining a relevant reaction coordinate is very crucial. To follow the minimum energy path, the $\mathrm{O} 1 \mathrm{HI}$ distance (see Fig. I for atomic numbering scheme) is defined as a reaction coordinate and the rest of the molecule is optimized along the proton transfer path in the $S_{0}$ and $S_{1}$ states without any symmetry constraints. The geometry optimization has been performed at fixed distance of the O1HI bond using the B3LYP/6-31G(d,p) methods for the $\mathrm{S}_{0}$ state, whereas the CIS/6-31G(d,p) method has been employed for the $S_{1}$ state. The $\mathrm{O} 1 \mathrm{HI}$ distance has been varied from $0.6 \AA$ to $2.0 \AA$ to construct the ground and excited state intramolecular proton transfer curves (GSIPT and ESIPT curves, respectively). From the test of the calculational methods used in the minimum energy path optimization of the $S_{1}$ state, it has been demonstrated by Sobolewski and Doncke that the CIS optimized reaction path is acceptable as far as the qualitative description of the potential energy profile is concemed. ${ }^{19.20}$ Thus it seems reasonable to assume that the CIS potential energy curve will follow the minimum energy paths of the proton transfer processes at least in between the enol and keto forms of two tautomers. For the calculation of the excited state potential energy curve, only the $\pi \pi^{*}$ state has been considered for all the title molecules since the next higher singlet state has been located significantly higher than the $\mathrm{S}_{1}\left(\pi \pi^{*}\right)$ state.

The shapes of the potential energy curves that describe the displacement of the hydrogen atom along the reaction coordinate in the ground and excited states play central roles to deternine photophysical and photochemical properties of this type of molecules. As mentioned above, the $a b$ initio methods that lack dynamical electron correlations (for example, (IS and CASSCF) give too high excitation energies and are not accurate enough to obtain reliable potential energy functions along the reaction path, even though the CIS method was demonstrated to provide a qualitatively correct characterization of the ESIPT processes in some cases. To obtain improved energies, TDDFT method with the $6-31 \mathrm{G}(\mathrm{d}, \mathrm{p})$ basis set has been employed. Vertical excitation energies of the $\pi \pi^{*}$ state has been calculated at the $\mathrm{S}_{0}$ geometry optimized with the B3LYP and MP2 methods, while the potential energy profile for the ESIPT process has been obtained by calculating TDDFT single point energy at the CIS optimized $S_{1}$ state geometry along the proton transfer reaction coordinate. Frack-Condon fluorescene energy is calculated at the minimum of the TDDFT curve in the $\mathrm{S}_{1}$ state. The $S_{0}$ state potential energy curve along the proton transfer coordinates were calculated using the B3LYP/6$31 \mathrm{G}(\mathrm{d}, \mathrm{p})$ method. In case that there exists a barrier along the proton transfer path in the $S_{1}$ state, the transition state has been located using the STQN method implemented in the Gaussian program by Schlegel et $a l^{32.33}$ to calculate the barrier height. All located transition states exhibit an expected imaginary normal frequency with a transition vector that corresponds to the motion of atoms associated with the proton transfer process.

\section{Results and Discussion}

CIS structures and potential profiles. The geometry parameters of the electronic ground state of the most stable conformers (enol form) of SA and its derivatives optimized at MP2/6-31G(d,p) and B3LYP/6-31G(d,p) levels are presented in Table 1 . Only those geometrical parameters which show significant changes and are regarded as important in the course of the proton transfer process are listed. In the electronic ground state, only the enol form of two tautomers is tumed out to be a stable structure on the $S_{0}$ potential energy surface along the reaction path at MP2 and B3LYP levels (vide infra). The molecules show planar structures at both calculational levels where the substituent groups, i.e., $-\mathrm{NH}_{2}$ and $-\mathrm{OCH}_{3}$ groups, lie on the plane of the aromatic ring.

In contrast to the ground state, for all the title molecules, two separate stable structures are found on the potential energy surface of the $S_{1}\left(\pi \pi^{*}\right)$ excited electronic state at the 
Table 1. Optimized geometry parameters (bond lengths in $\hat{\Lambda}$, bond angles in degrecs) and encrgies (in atomic units) of the $S_{0}$ state calculated at the MP2 and B3LYT levels, and of the stationary points on the potential energy surface of the $S_{1}$ state calculated at the level of CIS method

\begin{tabular}{|c|c|c|c|c|c|c|c|c|}
\hline \multirow[t]{3}{*}{ Parameters } & \multicolumn{8}{|c|}{$S_{1}$ state $^{a}$} \\
\hline & \multirow{2}{*}{$\begin{array}{c}\text { B3LYP } \\
\text { Enol }\end{array}$} & \multirow{2}{*}{$\begin{array}{l}\text { MP2 } \\
\text { Enol }\end{array}$} & \multirow{2}{*}{$\begin{array}{c}\text { CIS } \\
\text { Enol }\end{array}$} & \multicolumn{5}{|c|}{$\mathrm{TDDFT}^{\hbar}$} \\
\hline & & & & TS & Keto & Enol & TS & Keto \\
\hline \multicolumn{9}{|l|}{ SA } \\
\hline 0102 & 2.612 & 2.646 & 2.569 & 2.341 & 2.541 & 2.419 & 2.340 & 2.404 \\
\hline $\mathrm{OlH} 1$ & 0.987 & 0.979 & 0.972 & 1.196 & 1.672 & 1.100 & 1.300 & 1.450 \\
\hline $\mathrm{O} 2 \mathrm{H} 1$ & 1.728 & 1.775 & 1.694 & 1.189 & 0.977 & 1.378 & 1.087 & 1.019 \\
\hline $\mathrm{ClC} 2 \mathrm{Ol}$ & 122.81 & 123.60 & 119.48 & 117.02 & 118.89 & 117.69 & 117.17 & 117.85 \\
\hline $\mathrm{C} 2 \mathrm{ClC} 7$ & 118.58 & 118.89 & 118.91 & 115.84 & 118.70 & 116.88 & 115.86 & 116.78 \\
\hline $\mathrm{ClC} 7 \mathrm{O} 2$ & 124.50 & 124.80 & 124.71 & 122.12 & 124.92 & 123.16 & 122.02 & 122.87 \\
\hline Energies & -496.068223 & -494.652109 & -493.011652 & -493.003082 & -493.010337 & -495.916156 & -495.914730 & -495.914947 \\
\hline \multicolumn{9}{|l|}{$3 \mathrm{ASA}$} \\
\hline 0102 & 2.598 & 2.633 & 2.595 & 2.342 & 2.566 & 2.440 & 2.333 & 2.461 \\
\hline $\mathrm{OlH} 1$ & 0.988 & 0.981 & 0.967 & 1.195 & 1.701 & 1.090 & 1.24000 & 1.540 \\
\hline $\mathrm{O} 2 \mathrm{H} 1$ & 1.714 & 1.761 & 1.736 & 1.191 & 0.973 & 1.414 & 1.13711 & 0.997 \\
\hline $\mathrm{ClC2O1}$ & 123.54 & 124.21 & 121.65 & 118.97 & 121.62 & 119.77 & 119.04582 & 120.69 \\
\hline $\mathrm{C} 2 \mathrm{ClC} 7$ & 117.86 & 118.21 & 119.27 & 115.67 & 118.80 & 117.05 & 115.54983 & 117.32 \\
\hline $\mathrm{ClC} 7 \mathrm{O} 2$ & 124.61 & 124.90 & 124.82 & 121.92 & 124.68 & 123.26 & 121.72445 & 123.18 \\
\hline Energies & -551.426994 & -549.858954 & -548.059706 & -548.049978 & -548.058185 & -551.299357 & -551.297923 & -551.299007 \\
\hline \multicolumn{9}{|l|}{$5 \mathrm{ASA}$} \\
\hline 0102 & 2.621 & 2.653 & 2.596 & 2.345 & 2.506 & 2.519 & - & - \\
\hline $\mathrm{OlH1}$ & 0.985 & 0.978 & 0.965 & 1.246 & 1.613 & 1.050 & - & - \\
\hline $\mathrm{O} 2 \mathrm{H} 1$ & 1.743 & 1.786 & 1.735 & 1.145 & 0.987 & 1.551 & - & - \\
\hline $\mathrm{ClC} 2 \mathrm{Ol}$ & 123.42 & 124.01 & 120.14 & 117.51 & 119.01 & 119.20 & - & - \\
\hline $\mathrm{C} 2 \mathrm{ClC} 7$ & 118.47 & 118.83 & 119.41 & 115.94 & 118.26 & 118.31 & - & - \\
\hline $\mathrm{ClC} 7 \mathrm{O} 2$ & 124.60 & 124.89 & 125.24 & 122.15 & 124.30 & 124.47 & - & - \\
\hline Energies & -551.423045 & -549.854713 & -548.064311 & -548.050897 & -548.054250 & -551.299131 & - & - \\
\hline \multicolumn{9}{|l|}{$5 \mathrm{MeOSA}$} \\
\hline 0102 & 2.617 & 2.649 & 2.587 & 2.343 & 2.515 & 2.509 & - & - \\
\hline $\mathrm{OlH} 1$ & 0.986 & 0.979 & 0.968 & 1.232 & 1.628 & 1.050 & - & - \\
\hline $\mathrm{O} 2 \mathrm{H} 1$ & 1.738 & 1.781 & 1.721 & 1.157 & 0.984 & 1.539 & - & - \\
\hline $\mathrm{ClC} 2 \mathrm{O} 1$ & 123.37 & 124.04 & 119.79 & 117.23 & 118.86 & 118.83 & - & - \\
\hline $\mathrm{C} 2 \mathrm{C} 1 \mathrm{C} 7$ & 118.47 & 118.76 & 119.22 & 115.91 & 118.35 & 118.13 & - & - \\
\hline $\mathrm{ClC} 7 \mathrm{O} 2$ & 124.60 & 124.89 & 124.99 & 122.12 & 124.49 & 124.23 & - & - \\
\hline Energies & -610.590845 & -608.855280 & -606.904657 & -606.892816 & -606.897081 & -610.453580 & - & - \\
\hline
\end{tabular}

"6-31G(d,p) basis set is used for all calculations. ${ }^{b} \mathrm{CIS}$ optimized geonetry parameters and energies at the TDDFT minima and TS in the $\mathrm{S}_{1}$ state

CIS level corresponding to enol and keto tautomeric forms, respectively. Two tautomeric structures agree well with the previously reported CIS results on SA. In addition, the transition state between two minima, which can be characterized as the formation of $\mathrm{H}$-chelate ring, has been located at the CIS level. The transient structure has been verified as a saddle point for the ESIPT reaction by calculating the Hessian at the optimized geometry of the saddle point structure. A single imaginary vibrational frequency corresponding to the transfer of $\mathrm{H}$ atom between the hydroxyl and carbonyl oxygen atoms (O1 and $\mathrm{O} 2$, respectively) was calculated, which is consistent with the selection of intramolecular reaction coordinate for the proton transfer process. Thus the transition state structure optimized at a fixed distance of $\mathrm{O} 1 \mathrm{H} 1$ is believed to be very close to the saddle point at the level of CIS calculation. The CIS optimized geometry parameters of the transition state as well as the enol and keto forms in the $\mathrm{S}_{1}$ state are also presented in Table 1.

The CIS potential energy curve along the reaction coordinate of proton transfer in the $\mathrm{S}_{1}\left(\pi \pi^{3}\right)$ state is plotted in the upper panel in Figure 2. Two enol-keto tautomeric forms of SA on the $S_{1}$ potential energy surface are almost isoenergetic with the energy difference of $0.8 \mathrm{kcal} / \mathrm{mol}$, and separated by a significant barrier of about $5 \mathrm{kcal} / \mathrm{mol}$. As going from SA to $3 \mathrm{ASA}, 5 \mathrm{ASA}$, and $5 \mathrm{MeOSA}$, the tautomeric reactions in the $S_{1}$ state become more endothermic at the CIS level with the barrier heights changing from 5 to $6,8,7 \mathrm{kcal} / \mathrm{mol}$, respectively. The variations of the distance of the transferring $\mathrm{H}$ atom to the carbonyl oxygen $(\mathrm{O} 2 \mathrm{H} 1)$ and the distance between two oxygen atoms (O1O2) along the reaction coordinate are plotted in the middle panel in Figure 2. The optimized structures of the transition states at the CIS level for the title molecules exhibit that the distance between two 

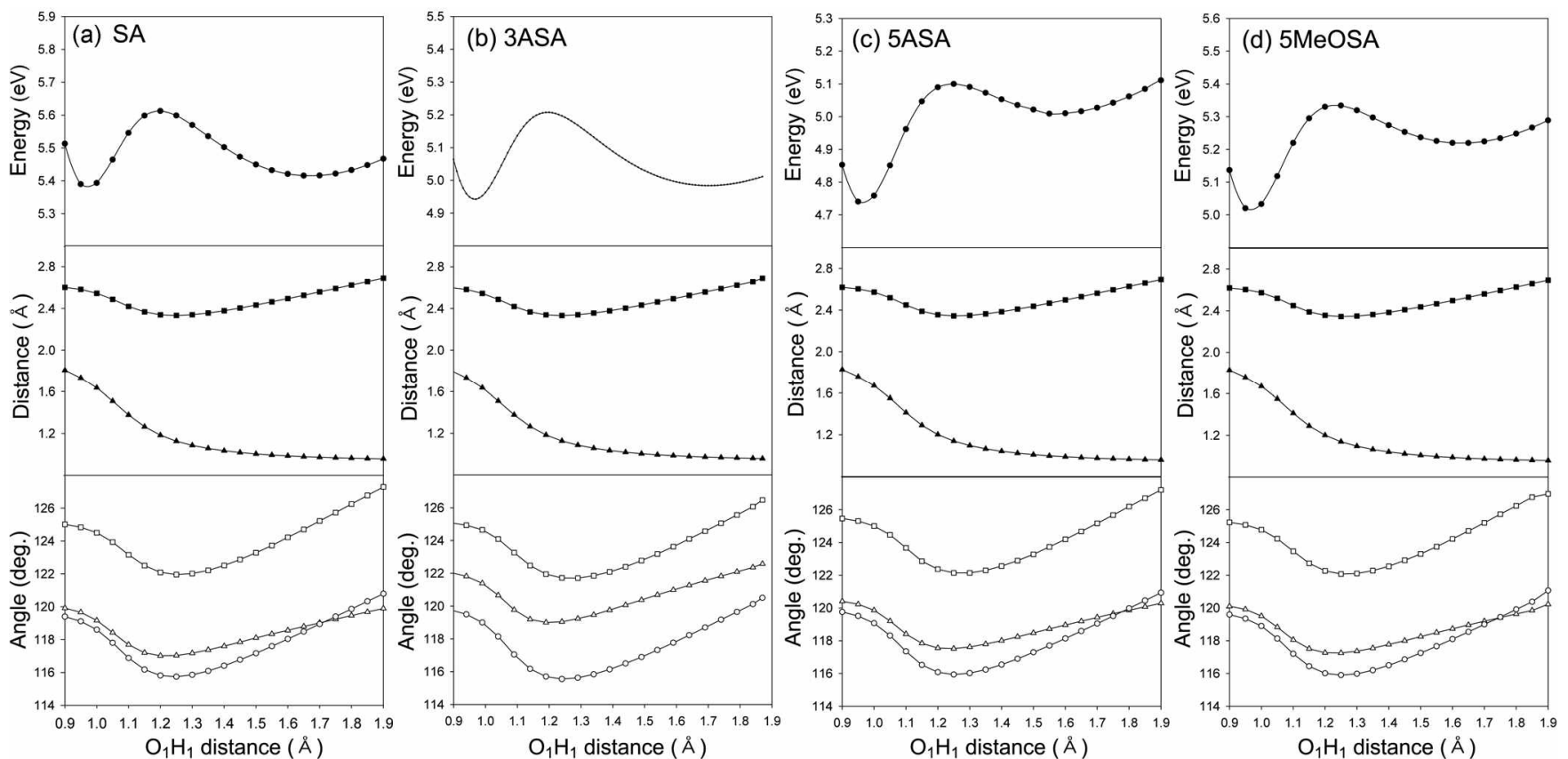

Figure 2. (upper panel) The CIS potential energy curve along the reaction coordinate of the proton transfer in the $\mathrm{S}_{1}\left(\pi \pi^{*}\right)$ state. (middle panel) Variations of the $\mathrm{O} 2 \mathrm{H} 1$ and $\mathrm{O} 1 \mathrm{O} 2$ distances along the reaction coordinate. (lower panel) Variations of the relevant bond angles which contribute to the variation of $\mathrm{O} 1 \mathrm{O} 2$ bond distance in the course of the proton transfer process: $\mathrm{C} 2 \mathrm{C} 1 \mathrm{C} 7$ (circles), $\mathrm{C} 1 \mathrm{C} 2 \mathrm{O} 1$ (triangles), CIC 702 (squares) för (a) $\mathrm{S} \Lambda$, (b) $3 \Lambda \mathrm{S} \Lambda$, (c) $5 \Lambda \mathrm{S} \Lambda$, (d) $5 \mathrm{McOS} \Lambda$

oxygen atoms $(\mathrm{O} 1 \mathrm{O} 2)$ in the $\mathrm{H}$-chelate ring gets significantly shorter (about $0.3 \AA$ ) compared with that in each tautomeric form. As a result, it is clear from the position of the transition state that the new $\mathrm{O} 2 \mathrm{HI}$ bond starts to be formed at relatively early stage of the $\mathrm{O} 1 \mathrm{H} 1$ bond elongation $(\triangle \mathrm{R}(\mathrm{OlH} 1)-0.3 \AA)$. Figure 2 clearly shows that the variations of $\mathrm{O} 1 \mathrm{O} 2$ and $\mathrm{O} 2 \mathrm{H} 1$ distances along the proton transfer coordinate are almost exactly same for all the title molecules, while the potential energy functions vary substantially from molecule to molecule. This result strongly implies that the photophysics of SA and its derivatives should be treated in terms of the structural change of the whole molecule rather than the local proton transfer between the phenolic $\mathrm{OH}$ group and the carboxyl group. The variations of the relevant bond angles which contribute to the variation of the $\mathrm{O} 1 \mathrm{O} 2$ and $\mathrm{O} 2 \mathrm{HI}$ bond distance are also given in the lower panel in Figure 2. Except for the paralle] shift of $\mathrm{ClC} 2 \mathrm{OI}$ angle by $2^{\circ}$ in case of 3ASA probably due to the interaction with the nearby amino group, the angle changes in the $\mathrm{H}$-chelate ring along the reaction coordinate are also almost same for all the molecules considered in this work. Apparently, a concerted skeletal relaxation of the Hchelate ring and the electronic effect of the substituents seem to be involved in the ESIPT process as has been suggested in the previous studies on SA. The other geometry parameters involving the aromatic ring and the substituent group did not show any significant changes as the $\mathrm{O} 1 \mathrm{HI}$ bond elongates along the reaction path.

Concluding the subsection, it can be said that, with the choice of the $\mathrm{O} 1 \mathrm{HI}$ distance as the minimum energy path for the proton transfer process, all the title molecules show very similar structural changes along the reaction coordinate. In other words, the title molecules under consideration experience the skeletal relaxation of the $\mathrm{H}$-chelate ring in common during the proton transfer without any further significant structural changes in the remaining parts of the molecules. Accordingly, it seems reasonable to assume that the variation of the photophysical properties of the title molecules is primarily due to the electronic effects of the substituent group, which is reflected in the shape of the potential energy function in the excited state along the reaction coordinate. As can be seen in Figure 2, the CIS method predicts very distinct double minimum potential energy profile in the excited state with a significant barrier for each of the title molecules. This result is, however, inconsistent with the experimental observations obtained in the spectroscopic study of solution or gas phase jet cooling of the molecules as pointed out by many research groups especially for SA. ${ }^{6.89 .11 .12 .19}$ If the barrier does exist and the excited state tautomerization occurs via tunneling through an excited state barrier between two excited state minima, the Stokes-shifted emission should also result from turneling through the barrier. Then the excited state proton transfer results in rather slow process. Moreover the intensity of the Stokes-shifted emission will increase with excitation energy since the tunneling rates increase with increasing excitation energy. The observed increase in the intensity of uv emission of SA as a function of excitation energy strongly implies that the ESIPT process in SA and its derivatives like the title molecules is associated with the intramolecular vibrational relaxation on the single minimum potential energy surface.

TDDFT potential energy functions. As stated above, 
even though the CI singles electronic structure method is believed to give an acceptable minimum energy path for the ESIPT process, the method is known to give too high excitation energies and is not accurate enough to obtain reliable potential energy functions along the reaction path. For the construction of improved potential energy profile for the proton transfer, single-point energy calculations at the CIS optimized geometries along the reaction coordinate have been performed using the TDDFT method to incorporate electron correlation effects for the excited state. The TDDFT potential energy profiles in the $S_{1}$ state along the proton transfer coordinate for the title molecules are presented in Figure 3 along with the CIS profiles for comparison. The CIS geometry parameters at the TDDFT minima and $\mathrm{TS}$ in the $\mathrm{S}_{1}$ state are also listed in Table 1. Al] the relevant energies of the $S_{0}$ and $S_{1}$ states were calculated relative to the energy of the ground state minimum optimized at the MP2/6-31G(d,p) level.

The potential energy profiles for the ground state intramolecular proton transfer (GSIPT) along the reaction coordinate were calculated using the B3LYP/6-31G(d,p) method, and also presented in Figure 3. For each title molecule, the $S_{0}$ state potential energy profile along the reaction path shows a relatively deep single minimum at the enol structure of the tautomers. The MP2 optimized ground state minimum occurs at $0.979,0.981,0.978$, and 0.979 for SA, 3ASA, 5ASA, and 5MeOSA, respectively. The potential energy increases monotonically and flattens at the $\mathrm{O} 1 \mathrm{HI}$ distance of around $1.3 \AA$. The GSIPT curves of the title molecules turned out to resemble each other very closely, which means that the differences in photophysical properties of the title molecules mainly determined by the variation of the ESIPT profiles along the reaction coordinate.

The excited state TDDFT potential energy profile of SA (Fig. 3a) resulted in a very flat function of the $\mathrm{OH}$ distance in the range of $\mathrm{R}_{\text {OI }}=1.0-1.6 \AA$, in contrast to the relatively deep single minimum potential energy function in the ground electronic state. Potential energy function with a smooth and shallow minimum for $\mathrm{SA}$ is already reported by Sobolewski and Domcke, ${ }^{20}$ in which the same TDDFT single point energy calculation at the CIS optimized geometry was performed using the same $6-31 \mathrm{G}(\mathrm{d}, \mathrm{p})$ basis set. They obtained a very shallow single minimum around the $\mathrm{OlH} 1$ distance of $1.1 \AA$ and $\mathrm{O} 2 \mathrm{H} 1$ distance of $1.3 \AA$. In their another work, it has been predicted that the potential energy surface of the $S_{1}$ state of SA is an extremely flat function of the $\mathrm{O} 1 \mathrm{HI}$ distance in the range of $1.0-1.5 \AA$, and varies by less than 0.5 $\mathrm{kcal} / \mathrm{mol}$. In contrast to the single minimum they observed in the TDDFT potential energy profile for SA, however, a close examination of the potential energy curve in Figure $3 \mathrm{a}$ reveals that there exist two minima corresponding to enoland keto-like tautomers at the OIH1 distance of $1.1 \AA$ and $1.45 \AA$, respectively. The barrier height is calculated to be about $0.9 \mathrm{kcal} / \mathrm{mol}\left(0.039 \mathrm{eV}, 315 \mathrm{~cm}^{-1}\right)$. Even though the barrier height is small enough compared with the accuracy of the TDDFT method and the zero-point vibrational energy of $\mathrm{OH}$ stretching, the size of the barrier height is still rather too high to overcome via thermal activation at room temperature $(k \mathrm{~T} \sim 0.6 \mathrm{kcal} / \mathrm{mol})$. The CIS optimized geometry parameters and energies at the TDDFT minima and TS in the $S_{1}$ state are presented in Table 1.

This discrepancy between the previous study and the present work may arise from the preclusion of the $C_{S}$ symmetry constraint for CIS geometry optimization of SA at fixed $\mathrm{O} 1 \mathrm{HI}$ distance. Taking into consideration of the error of CIS method for the choice of minimum energy path optimization, we don't see any reason for inclusion of the strict symmetry constraint during the geometry optimization along the reaction path. To confirm the origin of this discrepancy, however, we recalculated the ESIPT curve for SA with the $C_{S}$ symmetry constraint during geometry optimization. However, we obtained exactly same results for
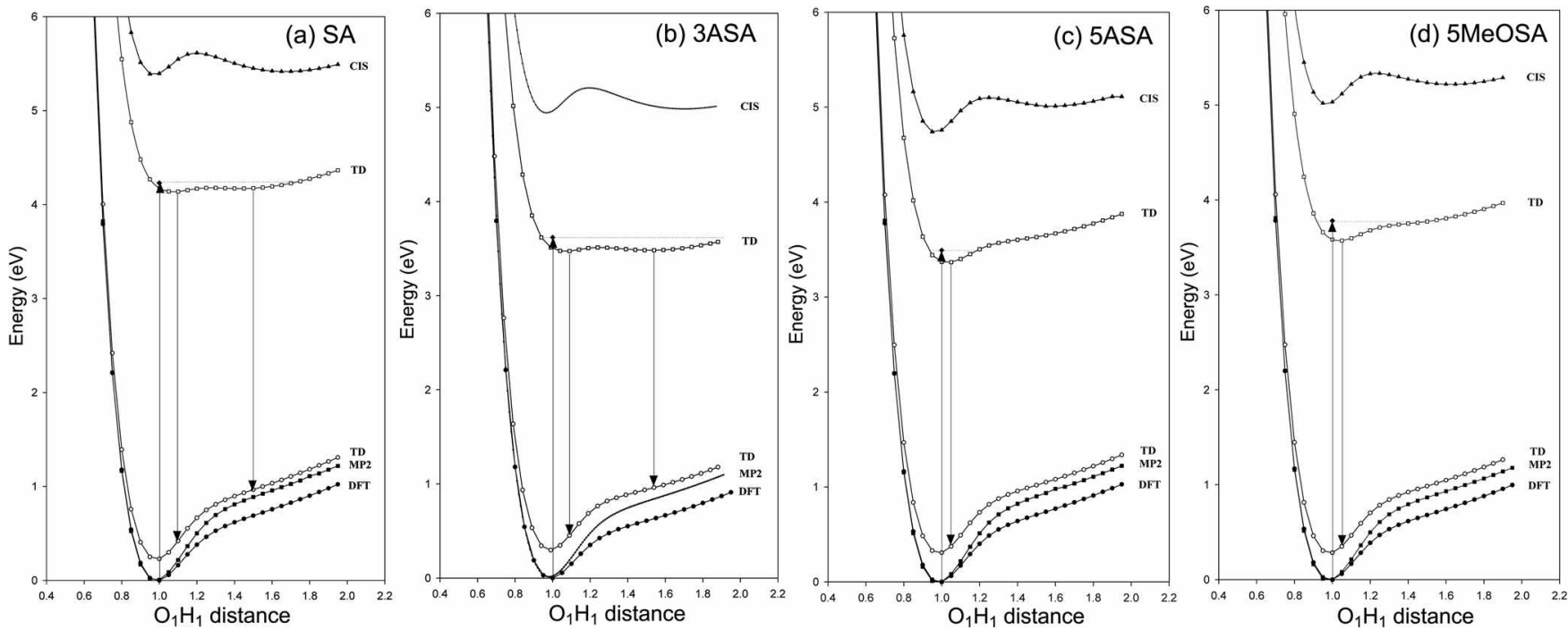

Figure 3. TDDFT potential energy profiles of the $S_{1}$ (solid squares) and $S_{0}$ states (solid circles) of (a) $S A$, (b) 3ASA, (c) 5ASA, and (d) $5 \mathrm{MeOS} \Lambda$ along the CIS-optimized reaction coordinates for the proton transfer processes in comparison with the CIS potential profiles (open squares). Ground state intramolecular proton transfer curves optimized at the B3LYP/6-3IG(d,p) level (open circles) are also presented. 
both calculations, i.e., two minima at the $\mathrm{O} 1 \mathrm{H} 1$ distances of 1.1 to $1.45 \AA$ with the same barrier height. The reason that they missed the second minimum on the excited state potential energy profile of SA is probably due to the use of sparser grid of points along the reaction coordinate. If the existence of the barrier is not real, it could be tentatively concluded from the shape of potential energy function that TDDFT method underestimates the stabilization of the potential energy profile near the barrier predicted by the CIS method, while the CASPT2 method overestimates it as pointed out by Sobolewski and Domcke. ${ }^{1920}$ Previous studies on the TDDFT adiabatic excitation energies show that, even though the TDDFT method improves upon the CIS method, the error is still far from the acceptable chemical accuracy of about $0.05 \mathrm{eV}$. The flat potential energy function of the $S_{1}$ state of SA, however, seems to agree qualitatively with the broad and large Stokes shift of SA (vide infra). With the exception of the small barrier, the excited state potential energy curve of SA obtained from TDDFT single point energy calculation reveals a very flat but slowly rising function along the proton transfer reaction coordinate in the range of $\mathrm{R}_{\mathrm{OIH}}=1.2-1.5 \AA$. The CASPT2 method with the aid of the CIS method, which is known to give more accurate energy values than TDDFT method, seems to reproduce a single minimum potential in the excited state even though the method shows an artifactual kink on the potential energy profile as reported by Sobolewski and Domcke. ${ }^{20}$ It should be pointed out, however, that it is still not clear that the complete removal of the potential energy barrier separating two tautomeric forms of SA in the $S_{1}$ state by this perturbative method is real or artifactual. A similar behavior has been observed for the ESIPT curve for malonaldehyde (MA), where the TDDFT method shows a small barrier of $-10 \mathrm{~cm}^{-1}$ while the CASPT2 method gives a single minimum along the proton transfer coordinate.

TDDFT potential energy profile of 3ASA (Fig. 3b) shows very similar structure compared to that of SA with about same small barrier height $(0.9 \mathrm{kcal} / \mathrm{mol})$, which is also negligible in the error range of the computational methods. TDDFT energy of keto form, however, has been lowered significantly giving almost the same energy compared with that of enol form $(\Delta \mathrm{E}=0.2 \mathrm{kcal} / \mathrm{mol})$. Deciding only from the flatter shape of the potential energy function, it can be predicted that 3ASA also show as large stokes shift as SA, and even more, although it depends on the reality of the existence of barrier between the enol and koto tautomeric forms of 3ASA. The flatness of the potential energy curve for SA and 3ASA in the excited state indicates that the large Stokes shift arises primarily from the increase of the ground state energy as the $\mathrm{O} 1 \mathrm{H} 1$ bond elongates along the reaction path. This, in turn, implies that the proton dislocation could be much larger than that predicted by the CIS calculation. The main difference observed in the excited state potential energy profiles between SA and 3ASA is the difference in lowering the excited state energies through TDDFT single point energy calculations. In case of SA, the TDDFT calculation lowers the CIS energy by $\sim 1.2 \mathrm{eV}$, while the energy is lowered by as much as $\sim 1.4 \mathrm{eV}$ in case of 3ASA. Unfortunately, to the best of our knowledge, we could not find any experimental data for 3ASA to compare with the calculated ones. Spectroscopic studies involving the excited state of 3ASA, however, will serve as another good test for the reliability of TDDFT method in the calculation of potential energy function of the excited state proton transfer process.

On the other hand, the TDDFT potential energy function of 5ASA in the $\mathrm{S}_{1}$ state (Fig. 3c) reveals a clear single minimum at around $\mathrm{R}_{01151}=1.05$, which is very close to the CIS optimized enol structure $\left(\mathrm{R}_{\mathrm{OlH}}=0.965 \AA\right.$ ). The potential function rises rather steeply and shows qualitatively different structures compared with the flat potential forms of SA and 3ASA. Substitution of the electron donating amino group at para position relative to the phenolic hydroxyl group definitely suppresses the proton transfer in the excited state, while the substitution at ortho position hardly affects the process. In case of $3 \mathrm{ASA}$, the amino group at ortho position to phenolic $\mathrm{OH}$ seems to even facilitate the proton process by stabilizing the keto structure even more. The $\mathrm{S}_{1}\left(\pi \pi^{\circ}\right)$ state potential energy curve (Fig. 3d) of 5MeOSA calculated at the TDDFT level along the CIS-optimized reaction coordinate shows similar behavior compared with that of 5ASA. The dispersed fluorescence spectra of jet cooled SA and 5MeOSA have been reported by Lahmani and Zehnacker-Rentien, ${ }^{8}$ and by N. Mikami groups. ${ }^{11} \mathrm{~A}$ broad and large Stokes shift with a maximum at $430 \mathrm{~nm}$ is observed in the spectrum of SA, which is attributed to the characteristic feature of the excited state proton dislocation. On the other hand, the Stokes shift of 5MeOSA is reduced compared with that of SA where the fluorescence center appeared at around $380 \mathrm{~nm}$. Moreover, the width of the dispersed fluorescence spectra of 5MeOSA turned out to be much narrower in comparison with that of SA. This substantial suppression of the excited state proton dislocation and the substitution effect of the methoxy group in the para position with respect to phenolic $\mathrm{OH}$ agree qualitatively well with the $S_{1}$ state TDDFT potential energy profile.

Figure $3 \mathrm{c}$ and Figure $3 \mathrm{~d}$ show that the TDDFT energy minimum in the excited state occurs at 1.05 and $1.05 \AA$ for 5 ASA and $5 \mathrm{MeOSA}$, respectively. Those are shifted only by $\sim 0.07 \AA$ relative to the MP2 optimized ground state minima occurring at 0.978 and $0.979 \AA$ for 5ASA and 5MeOSA, respectively. Other than that, to a good approximation, the TDDFT potential energy profile in the excited electronic states are parallel to that in the ground state along the proton transfer coordinate for 5ASA and 5MeOSA. This strongly implies that the pattern of the emission spectrum basically forms a mirror image of the absorption spectrum. Actually, Lahmani and Zehnacker-Rentien observed in the high resolution experiment of jet-cooled 5MeOSA that the dispersed fluorescence spectrum resulting from the $0-0$ level excitation exhibits a mirror image relationship with the fluorescence excitation spectrum with a strong band origin. The strong band origin observed in 5MeOSA forms a marked contrast with the small band origin of SA relative to other vibrational bands in the spectra. This experimental 
observation can be attributed the fact that the structure of $5 \mathrm{MeOSA}$ in the excited state is basically similar to that in the ground state, while that of SA changes considerably upon electronic excitation. In this regard, it is expected that 5ASA will also show a similar behavior with 5MeOSA in its absorption and emission properties.

TDDFT transition energies. From the discussions above, the TDDFT excited state potential energy profiles along the proton transfer reaction coordinate seem to give qualitatively correct answer for the trends observed in the experiments. For more quantitative discussions relevant to the proton transfer process, the transition energies involving absorption and fluorescence are evaluated. The TDDFT energies calculated at the stable points, i.e., enol, keto, and TS geometries, on the potential energy surfaces of the ground and the lowest excited singlet state of the title molecules are presented in Table 2. Similar notations and formats are used as those of Table 2 in ref. 20 for easy comparison. All the values are calculated relative to the energy minimum of the ground state optimized at the MP2/6-31G(d,p) level. Relevant spectroscopic transitions including vertical excitation, FranckCondon fluorescence, and spectral origin are illustrated in Figure 3.

Vertical excitation energy is calculated at the ground state minimum geometry optimized at the MP2/6-31G(d,p) level. The vertical energy of $S_{1}$ state calculated at the optimized geometry of $S_{0}$ state corresponds to the Franck-Condon excitation energy, which is associated approximately to the center of absorption band. The calculated TDDFT vertical excitation energies for SA, 3ASA, 5ASA, and 5MeOSA are listed in Table 2 in comparison with the available experimental values. The obtained TDDFT values of $4.23 \mathrm{eV}(\lambda=$ $293 \mathrm{~nm}$ ) for SA turned out to be too high by $0.33 \mathrm{eV}$, while the reported CASPT2 value of $3.92 \mathrm{eV}(\hat{\lambda}=316 \mathrm{~nm})$ is in good agreement with the experimental value of $3.9 \mathrm{eV}(\lambda=$ $310 \mathrm{~nm}$ ) observed for SA in cyclohexane. On the other hand, the TDDFT vertical excitation energy $(3.78 \mathrm{eV}, \hat{\lambda}=328 \mathrm{~nm})$ for $5 \mathrm{MeOSA}$ agrees a bit better with the experimental value of $3.65 \mathrm{eV}(\lambda=340 \mathrm{~nm})$ observed in cyclohexane in comparison with the case of SA. In contrast to the case of SA, however, the TDDFT method underestimates the vertical energy by $0.26 \mathrm{eV}$ compared with the experimental values observed in case of 5MeOSA. The energy level of the vertical excitation for each title molecule is plotted in Figure 3. It should also be noticed that, for SA and 3ASA, the excited state energy level that is reached by the $S_{1} \leftarrow S_{0}$ vertical transition is higher than the barrier separating the enol and keto tautomeric forms along the reaction coordinate. For 3ASA and 5ASA, the vertical excitation energies are calculated to be $3.62 \mathrm{eV}(\lambda=343 \mathrm{~nm})$ and $3.49 \mathrm{eV}(\lambda=$ $355 \mathrm{~nm}$ ), respectively.

The center of the fluorescence band ( $\varphi$ ) can be estimated from the TDDFT energy values presented in Table 2 and this value corresponds to the vertical transition from the TDDFT potential minima in the $S_{1}$ state to the ground state. The center of fluorescence due to the transition from the keto minimum in the $\mathrm{S}_{1}$ state is also listed in Table 2 for com- parison in case of SA and 3ASA. As mentioned above, the TDDFT potential energy profile shows very shallow double minima at $\mathrm{O} 1 \mathrm{Hl}=1.10$ and $1.45 \AA$ for $\mathrm{SA}$, and 1.09 and $1.54 \AA$ for 3ASA (see Table 1) with barrier height of about $350 \mathrm{~cm}^{-1}$. Those two minima nominally represent enol and keto structures along the proton transfer path in the excited state. Since the vibrational frequency of phenolic $\mathrm{OH}$ in the $\mathrm{S}_{1}$ state is estimated to be about $3,500 \mathrm{~cm}^{-1}$, even the zeropoint vibrational motion is more than enough to cover the whole range of the proton transfer coordinate. Moreover, setting aside the reality for the existence of barrier, the fact that the excited state energy level resulting from vertical excitation lies above the barrier thus makes it uncertain to assign the emitting structure to either enol or keto tautormers. It is anticipated that a competition between the fluorescence lifetime and the intramolecular vibrational relaxation time of the $S_{1}$ state is involved in determination of the emitting properties of the title molecules. TDDFT FranckCondon fluorescence energy from the enol form of SA (3.71 $\mathrm{eV}, \hat{\lambda}=334 \mathrm{~nm}$ ) is estimated too high by 0.91 and $0.81 \mathrm{eV}$ compared with the experimental values obtained in the solution and gas phase, respectively. On the other hand, if the transition from the keto form is assumed to be allowed, the obtained TDDFT values of $3.24 \mathrm{eV}(\lambda=383 \mathrm{~nm})$ for SA is in better agreement with the experimental value of $2.8 \mathrm{eV}$ $(\lambda=443 \mathrm{~nm})$ and $2.9 \mathrm{eV}(\lambda=428 \mathrm{~nm})$. The center of the fluorescence band from each of two tautomric forms of 3ASA is estimated in Table 2 to occur at about $411 \mathrm{~nm}$ and $492 \mathrm{~nm}$. In case of $5 \mathrm{MeOSA}$, where the potential energy profile shows a relatively deep single minimum, the calculated TDDFT fluorescence energy of $3.38 \mathrm{eV}(\lambda=367 \mathrm{~nm})$ is also agreement with the experimental value of $3.06 \mathrm{eV}(\hat{\lambda}$ $=400 \mathrm{~nm}$ ) obtained for $5 \mathrm{MeOSA}$ in cyclohexane, whereas it deviates by $0.23 \mathrm{eV}$ compared with that observed in the jetcooled gas phase experiment (Table 2). Similarly, the center of the fluorescence band for 5ASA has been evaluated as $2.99 \mathrm{eV}(\lambda=415 \mathrm{~nm})$.

The energy difference between the TDDFT potential energy minimum in the $S_{1}$ state and the MP2 optimized potential minimum in the $S_{0}$ state can be regarded as the $0-0$ line of the $S_{0} \leftrightarrow S_{1}$ transition. The calculated TDDFT $0-0$ line of the title molecules are estimated as $4.14,3.47,3.37$, and $3.57 \mathrm{eV}$ as listed in Table 2, where the corrections for the zero point vibrational energies are not included. The calculated TDDFT values of $3.57 \mathrm{eV}(\hat{\lambda}=347 \mathrm{~nm})$ for the 0 0 transition of $5 \mathrm{MeOSA}$ is in good agreement with the experimentally observed value of $3.49 \mathrm{eV}(\lambda=355 \mathrm{~nm})$. In other words, the TDDFT 0-0 transition energy is underestimated only by $0.11 \mathrm{eV}$ compared with the experimental value. On the other hand, in case of SA, the TDDFT method overestimates the $0-0$ transition energy by $0.45 \mathrm{eV}$ in comparison with experimental value (Table 2). Similarly, the TDDFT energies for the $0-0$ transition for 3ASA and 5ASA are estimated as $3.47 \mathrm{eV}(\hat{\lambda}=357 \mathrm{~nm})$ and $3.37 \mathrm{eV}(\hat{\lambda}=368$ $\mathrm{nm})$, respectively. It has been generally known from the previous work that the TDDFT method overestimates the relevant transition energies by about $0.5 \mathrm{eV}$ compared with 
Table 2. TDDFT energies of SA, 3ASA, 5ASA, and 5MeOSA calculated at the minimum of the $S_{0}$ and $S_{1}$ states relative to the minimum energy of the ground state (in $\mathrm{cV}$ ). The center of fluorescence $\left(v_{n}\right)$, and Stokes shift $(1)$ ) are given for comparison with experiments when available

\begin{tabular}{|c|c|c|c|c|c|c|}
\hline Geometry & State & & TDDFT (enol) & TDDFT (keto) & Experiment & Spectroscopic Quantities \\
\hline \multicolumn{7}{|l|}{ SA } \\
\hline$S_{0}$ & $S_{1}$ & $v_{3}$ & $4.23(293 \mathrm{~nm})$ & $4.23(293 \mathrm{~nm})$ & $3.9(318 \mathrm{~nm})^{a}$ & Franck-Condon absorption \\
\hline$S_{\mathrm{l}}$ & $\mathrm{S}_{0}$ & & $0.43\left(3.468 \mathrm{~cm}^{-1}\right)$ & $0.90\left(7,259 \mathrm{~cm}^{-1}\right)$ & & \\
\hline \multirow[t]{3}{*}{$S_{l}$} & $S_{1}$ & $v_{000}$ & $4.14(299 \mathrm{~nm})$ & $4.14(299 \mathrm{~nm})$ & $3.69(336 \mathrm{~nm})^{h}$ & $0-0$ band \\
\hline & & $v_{\mathrm{f}}$ & $3.71(334 \mathrm{~nm})$ & $3.24(383 \mathrm{~nm})$ & $2.8(443 \mathrm{~nm})^{a}, 2.9(428 \mathrm{~nm})^{b}$ & Franck-Condon fluorescence \\
\hline & & $v_{s}$ & $0.52\left(4.194 \mathrm{~cm}^{-1}\right)$ & $0.99\left(7,984 \mathrm{~cm}^{-1}\right)$ & $1.1\left(8,872 \mathrm{~cm}^{-1}\right)^{a}, 1.0\left(8,065 \mathrm{~cm}^{-1}\right)^{b}$ & Stokes shift \\
\hline \multicolumn{7}{|c|}{ 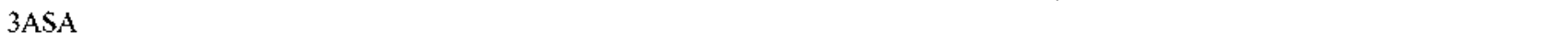 } \\
\hline $\mathrm{S}_{0}$ & $S_{1}$ & $v_{3}$ & $3.62(343 \mathrm{~nm})$ & $3.62(343 \mathrm{~nm})$ & - & Franck-Condon absorption \\
\hline$S_{l}$ & $\mathrm{~S}_{0}$ & & $0.45\left(3.629 \mathrm{~cm}^{-1}\right)$ & $0.95\left(7,662 \mathrm{~cm}^{-1}\right)$ & - & \\
\hline \multirow{3}{*}{$S_{l}$} & $S_{1}$ & $v_{0-0}$ & $3.47(357 \mathrm{~nm})$ & $3.47(357 \mathrm{~nm})$ & - & $0-0$ band \\
\hline & & $v_{\mathrm{f}}$ & $3.02(411 \mathrm{ntn})$ & $2.52(492 \mathrm{~nm})$ & - & Franck-Condon fluorescence \\
\hline & & $v_{s}$ & $0.60\left(4.838 \mathrm{~cm}^{-1}\right)$ & $1.10\left(8,872 \mathrm{~cm}^{-1}\right)$ & - & Stokes shift \\
\hline \multicolumn{7}{|l|}{ 5ASA } \\
\hline$S_{0}$ & $S_{1}$ & $v_{3}$ & $3.49(355 \mathrm{~nm})$ & - & - & Franck-Condon absorption \\
\hline$S_{l}$ & $\mathrm{~S}_{0}$ & & $0.38\left(3065 \mathrm{~cm}^{-1}\right)$ & - & - & \\
\hline \multirow[t]{3}{*}{$S_{l}$} & $S_{1}$ & $v_{0,0}$ & $3.37(368 \mathrm{~nm})$ & - & - & $0-0$ band \\
\hline & & $v_{\mathrm{f}}$ & $2.99(415 \mathrm{~nm})$ & - & - & Franck-Condon fluorescence \\
\hline & & $v_{s}$ & $0.50\left(4.051 \mathrm{~cm}^{-1}\right)$ & - & - & Stokes shift \\
\hline \multicolumn{7}{|l|}{ 5MeOSA } \\
\hline $\mathrm{S}_{0}$ & $S_{1}$ & $v_{3}$ & $3.78(328 \mathrm{~nm})$ & - & $3.65(340 \mathrm{~nm})^{c}$ & Franck-Condon absorption \\
\hline$S_{1}$ & $\mathrm{~S}_{0}$ & & $0.19\left(1.532 \mathrm{~cm}^{-1}\right)$ & - & $0.40\left(3,226 \mathrm{~cm}^{-1}\right)^{c}$ & \\
\hline \multirow[t]{3}{*}{$S_{l}$} & $S_{1}$ & $v_{000}$ & $3.57(347 \mathrm{~nm})$ & - & $3.49(355 \mathrm{~nm})^{d}$ & $0-0$ band \\
\hline & & $v_{\mathrm{f}}$ & $3.38(367 \mathrm{~nm})$ & - & $3.06(400 \mathrm{~nm})^{c}, 3.26(380 \mathrm{~nm})^{d}$ & Franck-Condon fluorescence \\
\hline & & $v_{s}$ & $0.40\left(3.226 \mathrm{~cm}^{-1}\right)$ & - & $0.59\left(4,758 \mathrm{~cm}^{-1}\right), 0.23\left(1.835 \mathrm{~cm}^{-1}\right)^{d}$ & Stokes shift \\
\hline
\end{tabular}

"ref. [34], "ref. [35], "ref. [36], "ref. [31]

more accurate CASPT2 method. The TDDFT results for 5MeOSA, however, reveal that the TDDFT method reproduces the experimental transition energies fairly well. Actually the transition energies for absorption and emission are systematically underestimated by less than $0.25 \mathrm{eV}$ in TDDFT.

The Stokes shifts $\left(v_{s}\right)$ of fluorescence bands of the title molecules, which are evaluated as the difference between the centers of absorption and fluorescence, are presented in Table 2. As expected from above discussions, SA and 3ASA show larger Stokes shift than 5ASA and 5MeOSA, which means that introduction of an electron donating group in the para position relative to the phenolic hydroxyl group suppresses the proton transfer in the excited state. In case of 5MeOSA, the TDDFT Stokes shift of $0.40 \mathrm{eV}\left(3,226 \mathrm{~cm}^{-1}\right)$ reproduce reasonably well the experimental values of 0.59 $\mathrm{eV}\left(4,758 \mathrm{~cm}^{-1}\right)$ and $0.23 \mathrm{eV}\left(1,835 \mathrm{~cm}^{-1}\right)$ observed for $5 \mathrm{MeOSA}$ in cyclohexane and jet-cooled gas phase, respectively. About same value of the Stokes shift $(0.50 \mathrm{eV}, 4,051$ $\mathrm{cm}^{-1}$ ) has been obtained for 5ASA. For both molecules, the $\mathrm{O} 1 \mathrm{H1}$ bond distances are calculated to be about $0.98 \mathrm{in}$ the MP2 optimized ground state and $1.05 \AA$ at the TDDFT minimum in the excited state (see Table 1 ). The relatively small elongation of the phenolic $\mathrm{OH}$ bond distance $(0.07 \AA)$ reflects the fact that the substitution of an electron donationg group at para position reduces the acidity of the hydroxyl group in the excited state as was first pointed out by Weller. On the other hand, the TDDFT Stokes shift of $0.52 \mathrm{eV}$ $\left(4,194 \mathrm{~cm}^{-1}\right)$ evaluated from the enol form of SA gives too low value by about $0.5 \mathrm{eV}$, while the Stokes shift of $0.99 \mathrm{eV}$ $\left(7,984 \mathrm{~cm}^{-1}\right)$ evaluated from the keto form is in good agreement with the experimentally observed values of $1.0 \mathrm{eV}$ $\left(8,065 \mathrm{~cm}^{-1}\right)$ and $1.1 \mathrm{eV}\left(8,872 \mathrm{~cm}^{-1}\right)$. The $\mathrm{O} 1 \mathrm{H1}$ distances at two TDDFT potential energy minima in the $\mathrm{S}_{1}$ state are calculated to be 1.10 and 1.45 corresponding to the enol and keto forms, respectively, while that in the ground state is found to be about 0.98 at the MP2 and B3LYP levels (see Table 1). In other words, the TDDFT method predicts that the elongation of the phenolic $\mathrm{OH}$ bond distance in the $\mathrm{S}_{1}$ state is about $0.12 \AA$ and $0.47 \AA$ for enol and keto emitting forms, respectively. Even though, assuming a single minimum at the $\mathrm{O} 1 \mathrm{H} 1$ distance of $1.1 \AA$, the CASPT2 potential energy profile seems to reproduce very closely the experimental transition energies and Stokes shift of SA, the result is somewhat uncertain due to the artifactual kink in the vicinity of potential energy minimum in the excited state as pointed out by Sobolewski and Domcke. ${ }^{20}$ As mentioned above, in case of SA, the TDDFT method gives systematically overestimated transition energies for absorption and emission by about $0.33 \mathrm{eV}$. However, if we assume the TDDFT potential energy profile is parallel to the CASPT2 profile as confirmed by Sobolewski and Domcke's work, the TDDFT method is 
expected to give a reliable result for the Stokes shift of SA. According to this reasoning and the extremely flat potential energy profile of SA in the excited state, we propose a possibility that the proton dislocation in the $S_{1}$ state could be much larger than the previously predicted value of $0.15 \AA$. It is not certain, however, that the small barrier separating the enol and keto tautomeric forms of SA in the excited state is real or not. From the parallel trends of the TDDFT and CASPT2 methods observed in other molecules like $o$-hydroxybenzaldehyde (OHBA), 7-hydroxy-1-indanone (7HIN), malonaldehyde (MA) as well as SA, it can be concluded qualitatively for now that the potential energy profile of SA in the $S_{1}$ state is an extremely flat function of the proton transfer reaction coordinate. For 3ASA, compared with SA, almost the same amount of the Stokes shift is predicted by the TDDFT method, i.e., $0.60 \mathrm{eV}\left(4,838 \mathrm{~cm}^{-1}\right)$ and $1.10 \mathrm{eV}$ $\left(8,872 \mathrm{~cm}^{-1}\right)$ from enol and keto emitting forms, respectively. Two TDDFT minima in the excited state tautomeric structures correspond to the $\mathrm{O} 1 \mathrm{H} 1$ distance of $1.09 \AA$ and $1.54 \AA$, respectively. With the same reasoning as in case of $\mathrm{SA}$, a large elongation of the phenolic $\mathrm{OH}$ bond is predicted upon photoexcitation.

\section{Conclusion}

The potential energy profiles for the excited state intramolecular proton transfer reaction have been calculated for SA, 3ASA, 5ASA, and 5MeOSA, employing the CIS and TDDFT methods. As in previous studies, the excited state potential energy functions of the title molecules calculated at the CIS level exhibit two distinct minima corresponding to enol and keto tautomeric forms separated by substantial energy barriers of about $5 \mathrm{kal} / \mathrm{mol}$. This result is, however, inconsistent with the experimental observations obtained in the spectroscopic study of solution or gas phase jet cooling of SA and 5MeOSA. To obtain more reliable potential energy profile and improved transition energies, the TDDFT single point energy calculation at the CIS optimized geometries along the reaction path has been perfonmed to incorporate electron correlation effects for the excited state.

The excited state TDDFT potential energy profiles of SA and 3ASA resulted in very flat function of the $\mathrm{OH}$ distance in the range of $\mathrm{R}_{O H}=1.0-1.6 \AA$, in contrast to the relatively deep single minimum potential energy function in the ground state. However, even though the barrier height is negligible $\left(\sim 350 \mathrm{~cm}^{-1}\right)$ in the error range of the calculational methods, we observed a double minimum potential energy profile along the proton transfer path in the $\mathrm{S}_{1}$ state instead of a single minimum profile reported in previous work on SA using the same method. It is not certain for now that the existence of the small barrier on the potential energy curves of SA and 3ASA in the $\mathrm{S}_{1}$ state is real or not. But the important point is that the overall shape of the potential energy profile in the exited state is so flat that it is hard to assign the emitting form strictly to either enol or keto form of the tautomers. On the other hand, the TDDFT potential energy function for 5ASA and 5MeOSA reveals a clear single minimum where the minimum is located close to the CIS optimized enol structure. The ESIPT curves of 5ASA and $5 \mathrm{MeOSA}$ turned out to resemble very closely the GSIPT curves along the reaction path, and show qualitatively different shapes compared with the flat potential forms of SA and 3ASA. Substitution of the electron donating group at para position relative to the phenolic hydroxyl group definitely suppresses the proton transfer in the excited state, while the substitution at ortho position does not substantially affect the process.

The transition energies associated with the ESIPT process including vertical absorption, Franck-Condon fluorescence, and spectral origin are estimated from the TDDFT potential energy profiles. Comparison of the estimated transition energies with the experimental ones indicate that the TDDFT method gives a qualitatively reliable shape of potential energy profile for the ESIPT process. The TDDFT method predicts, however, systematically overestimated transition energies in case of SA, while the method reproduces the experimental values observed for $5 \mathrm{MeOSA}$ reasonably well. From the reliability of the TDDFT method as discussed above, it can be concluded that the spectral properties of the title molecules seem to be characterized primarily by the vibrational relaxation on the single minimum potential surface in the excited state, and the extreme flatness of the potential profiles of SA and 3ASA along the proton transfer path may results in much larger proton dislocation in the $\mathbf{S}_{1}$ state than the previously predicted value of $0.15 \AA$. Further experimental spectroscopic studies will be directed to 3ASA, 5ASA as well as the hydrogen bonded complexes with $\mathrm{H}_{2} \mathrm{O}$.

Acknowledgements. This work was supported by Korean Research Foundation Grant (KRF-2002-015-CP0155), and partly by research program 2007 of Kookmin University in Korea.

\section{References}

I. Weller, A. Prog. React. Kinet. 1961, $I, 188$.

2. Weller, A. A. Nattrwissenchaflen $1995,42,175$.

3. Weller, A. Z. Electrochem, 1956, 60, 1144.

4. Goodman, J.; Brus, L. E. J. Am. Chem. Soc. 1978, 100, 7472.

5. Heimbrook, L.; Kenny, J. E.; Kohler, B. E.; Scou, G. W. J. Phys. Chem. 1983, 87, 280.

6. Yahagi, T.; Fujii, A.; Ebata, T.; Mikami, N. J. Phys. Chem. A 2001, 105, 10673 .

7. Herek, J. L.; Pedersen, S.; Banares, L.; Zewail, A. H. J. Chem. Phys, 1992, 97,9046.

8. Lahmani, F.; Zeknacker-Rentien, A. J. Phys. Chem, A 1997, 101, 6141.

9. Bisht, P. B.; Petek, H.; Yoshihara, K.; Nagashima, U. J. Chem. $P / h s .1995,103,5290$.

10. Heimbrook, L. A.; Kenny, J. E.; Kohler, B. E.; Scou, G W. $J$. Chem. Phys, 1981, 75, 5201.

11. Abou El-Nast, E. A.; Fujii, A.; Ebala, T.; Mikami, N. Chem. Phys. Leff. 2003, 376, 788 .

12. Abou El-Nasr, E. A.; Fujii, A.; Yahagi, T.; Ebata, T.; Mikami, N. J. Phss. Chem. A 2005, 109, 2498.

13. Wang, D. P.; Chen, S. G.; Chen, D. Z. J. Photochem. Photobiol. A: Chem. 2004, 162, 407. 
14. Banerjee, D.; Mukhopadhyay, M.; Mukherjee, S. J. Photochen. Photobiol. A. Chem. 2005, $172,250$.

15. Liang, Y. H.; Yi, P. G.; Cao, C. Z. J. Mol. Strwe- THEOCHEM $2005,729,163$.

16. Ahn, D. S.; Lee, S.; Kim, B. Chem. Phys. Lett. 2004, $390,384$.

17. Calalan, J.; Femandez-Alonso, J. J. J. Mol. Struc. 1975, 27, 59.

18. Orttung, W. H.; Scott, G. W; Vossoghi, D. J. Mol. Struct. 1984, 109,161 .

19. Sobolewski, A. L.; Domcke, W. Chem. Phys. 1998, 232, 257.

20. Sobolewski, A. L.; Domcke, W. Phys. Chem. Chem. Phys, 1999, 1,3065 .

2I. Vener, M. V.; Scheiner, S. J. Phys, Chem. 1995, 99, 642,

22. Zhang, W.; Shi, B.; Shi, J. J. Mol. Strte: THEOCHEM 2005, 73/, 219.

23. Casadesus, R.; Moreno, M.; Lluch, J. M. Chen. Phys. 2003, 290, 319.

24. Gong Z.; Lagowski, J. B. J. Mol. Struc: THEOCHEM 2005, 729, 211 .

25. Nosenko, Y.; Stepanenko, Y.; Wu, F.; Thummel, R. P.; Mordzinski, A. Chem. Phys. Letf. 1999, 315,87.

26. Liu, F.; Zuo, P.; Meng, L.; Zheng, S. J. J. Mol. Strik.THEOCHEM, 2005, 726, 161 .

27. Casadesus, R.; Vendrell, O.; Moreno, M.; Lluch, J. M. Chem. Phys. Lett. 2005, 405, 187.

28. Yi, P. G.; Liang, Y. H. Chem. Phys. 2006, $322,382$.

29. Sobolewski, A. L.; Domcke, W. J. Phys. Chen. A 2004, Jos, 10917.

30. Shukla, M. K.; Leszczynski, J. Int. J. Quan. Chen. 2005, J05, 387.

31. Abou El-Nast, E. A.; Fujii, A.; Ebata, T.; Mikami, N. Mol. Phys.
$2005,103,1561$.

32. Peng. C.; Ayala, P. Y.; Schlegel, H. B.; Frisch, M. J. J. Comp. Chen. 1996, 17, 49 .

33. Peng, C. Schlegel, H. B. Israel J. Chem. 1993, 33, 449.

34. Pant, D. D.; Joshi, H. C.; Bisht, P. B.; Tripathi, H. B. Chem. Phys. 1994, $185,137$.

35. Bisht, P. B.; Petek, H.; Yoshihara, K.; Nagashima, U. J. Chem. $P / n$ ss. 1995, 103, 5290.

36. Smoluch, M.; Joshi, H.; Gerssen, A.; Gooijer, C.; van der Zwan, G. J. Phys. Chent $A 2003,109,535$.

37. Frisch, M. J.; Trucks, G. W.; Schlegel, H. B.; Scuseria, G. E.; Robb, M. A.; Cheeseman, J. R.; Montgomery, J. A., Jr; Vreven, T.; Kudin, K. N.; Burant, J. C.; Millam, J. M.; Jyengar, S. S.; Tomasi, J.; Barone, V.; Mennucci, B.; Cossi, M.; Scalmani, G.; Rega, N.; Petersson, G. A.; Nakatsuji, H.; Hada, M.; Ehara, M.; Toyola, K.; Fukuda, R,; Hasegawa, J.; Ishida, M.; Nakajima, T.; Honda, Y.; Kitao, O.; Nakai, H.; Klene, M.; Li, X.; Knox, J. E; Hratchian, H. P.; Cross, J. B.; Bakken, V.; Adamo, C.; Jaramillo, J.; Gomperts, R.; Stratmann, R. E.; Yazyev, O.; Austin, A. J.; Cammi, R.; Pomelli, C.; Ochterski, J. W.; Ayala, P. Y.; Morokuma, K.; Voth, G. A.; Salvador, P.; Dannenberg, J. J.; Zakrzewski, V. G.; Dapprich, S.; Daniels, A. D.; Strain, M. C.; Farkas, O.; Malick, D. K.; Rabuck, A. D.; Raghavachari, K.; Foresman, J. B.; Orliz, J. V;; Cui, Q.; Baboul, A. G; Clifford, S.; Cioslowski, J.; Stefanov, B. B.; Liu, G.; Liashenko, A.; Piskorz, P.; Komaromi, I.; Martin, R. L.; Fox, D. J.; Keith, T.; Al-Laham, M. A.; Peng. C. Y.; Nanayakkara, A.; Challacombe, M.; Gill, P. M. W.; Johnson, B.; Chen, W.; Wong. M. W.; Gonzalez, C.; Pople, J. A. Gaussian 03, revision B, 05; Gaussian, Inc.: Wallingford, CT, 2003. 